\title{
3D Cardiac Segmentation Using Temporal Correlation of Radio Frequency Ultrasound Data
}

\author{
Maartje M. Nillesen ${ }^{1}$, Richard G.P. Lopata ${ }^{1}$, Henkjan J. Huisman², \\ Johan M. Thijssen ${ }^{1}$, Livia Kapusta ${ }^{3}$, and Chris L. de Korte ${ }^{1}$ \\ 1 Clinical Physics Laboratory, Department of Pediatrics \\ m.m.nillesen@cukz.umcn.nl \\ 2 Department of Radiology \\ 3 Pediatric Cardiology, Department of Pediatrics \\ Radboud University Nijmegen Medical Centre
}

\begin{abstract}
Semi-automatic segmentation of the myocardium in 3D echographic images may substantially support clinical diagnosis of heart disease. Particularly in children with congenital heart disease, segmentation should be based on the echo features solely since a priori knowledge on the shape of the heart cannot be used. Segmentation of echocardiographic images is challenging because of the poor echogenicity contrast between blood and the myocardium in some regions and the inherent speckle noise from randomly backscattered echoes. Phase information present in the radio frequency (rf) ultrasound data might yield useful, additional features in these regions. A semi-3D technique was used to determine maximum temporal cross-correlation values locally from the rf data. To segment the endocardial surface, maximum cross-correlation values were used as additional external force in a deformable model approach and were tested against and combined with adaptive filtered, demodulated rf data. The method was tested on full volume images (Philips, iE33) of four healthy children and evaluated by comparison with contours obtained from manual segmentation.
\end{abstract}

\section{Introduction}

Three-dimensional (3D) segmentation of the endocardial surface could be a helpful tool for clinical assessment of 3D echocardiographic images. Segmentation may not only serve as an important tool for assessment of cardiac output and other functional parameters but also as a preprocessing step for tissue characterization, strain imaging and diagnosis of congenital deformities. Since manual segmentation of the left ventricle in 3D ultrasound image sequences is timeconsuming and is subject to inter-expert variability, (semi-) automatic segmentation techniques are required (see [1] for an overview). The influence of speckle noise and the poor contrast in echogenicity between the blood and the heart wall in regions where the muscle fibers are mainly parallel to the propagation direction of the ultrasound beam, impose strong demands on the segmentation 
algorithm. Automatic segmentation purely based on differences in echogenicity will be problematic in these low echogenicity regions as the contrast between blood and myocardial tissue is absent. Therefore, a technique using temporal information might be beneficial.

Segmentation techniques using shape and appearance models of the left ventricle have been described [2] to overcome these segmentation problems. However, in children with congenital deformities of the heart, inclusion of a priori knowledge about the average shape of the left ventricle will lead to erroneous segmentation results. The addition of temporal information by using cross-correlation techniques might facilitate segmentation in these problematic low contrast regions. Few segmentation methods use the rich phase information available in the radio frequency (rf) signal 3, 4, 5. Yan et al. 6] propose the use of maximum correlation coefficients obtained from a phase sensitive speckle tracking method for segmentation of the left ventricle.

This study builds on the work of Yan et al. 6] and Nillesen et al. 7] using a combination of maximum cross-correlation values and adaptive mean squares (AMS) filter values as external forces of a gradient-based deformable simplex mesh model. The cross-correlation values were obtained from a semi-3D coarseto-fine displacement algorithm (developed for strain estimation). This combined method was compared with segmentation results using correlation or AMS values as an external force separately. All results were evaluated by comparing them with manual segmentation.

\section{Materials and Methods}

Echocardiographic image sequences of the left ventricle were obtained in four healthy children. Echographic imaging was approved by the local ethics committee and parents gave their informed consent for using the data. Transthoracic full volume image sequences (ECG-gated, volume rate $\approx 50 \mathrm{~Hz}$ ) were obtained in long/short axis views. Rf-data were acquired directly after receive beam-forming using an iE33 ultrasound system (Philips Medical Systems, Bothell, WA, USA), equipped with an rf-interface and a pediatric X7-2 matrix array transducer (2-7 $\mathrm{MHz}$ ). Rf-data were sampled at $16 \mathrm{MHz}$ and transmitted to an external hard disk. The data were band pass filtered (FIR least squares filter [2-5 MHz]) to prevent disturbance by clutter and noise from frequencies outside the frequency band of the transducer. For constructing echograms, the data were amplitude demodulated using the Hilbert transform method.

\subsection{Temporal Cross-Correlation of the Radio Frequency Signal}

As velocity of the blood flow is expected to be higher than velocity of the surrounding myocardial tissue, rf-signals will correlate less for fast moving blood than for myocardial tissue. Temporal correlations might thus be used as a feature for distinguishing between blood and the heart muscle. For 3D strain imaging purposes, the cross-correlation function $(\mathrm{CCF})$ of $2 \mathrm{D}$ windows of rf-data was 
calculated [8], 9] using two subsequent full volumes. The peak of the crosscorrelation function reveals the displacement of a $2 \mathrm{D}$ segment of rf-data in the next time frame in $2 \mathrm{D}$ space. A $2 \mathrm{D}$ coarse-to-fine displacement estimation algorithm was expanded into an iterative semi-3D approach. 2D reference windows of $50 \times 5$ (axial x lateral) samples within the axial-azimuth plane were matched with a search area of $150 \times 11$ samples in the next frame. Both the CCF and the axial and lateral displacements were estimated. Next, the axial displacements were used as an offset to estimate axial and elevational displacements and the $\mathrm{CCF}$ in the axial-elevational plane. Hence, the displacements in three orthogonal directions in 3D space were assessed. This semi-3D approach was preferred over a full-3D approach since the iterative 2D cross-correlation calculation imposed a lower computational load and no significant difference in performance and precision was found between both methods 9. The maximum cross-correlation (MCC) values were found for each window. Window overlap in the axial direction was $75 \%$, resulting in a cross-correlation image of $147 \times 62 \times 56$ samples. This corresponds to a pixel resolution of $600 \mu \mathrm{m}$ in the axial direction.

\subsection{D Adaptive Filtering}

Besides the maximum cross-correlation values, adaptive filtering was used as a more conventional method to optimize the distinction between blood and myocardium. 3D Adaptive Mean Squares filtering of the amplitude demodulated data was applied in the spatial domain. The 3D filter kernel size was related to image speckle size and contained approximately $5 \times 2 \times 2$ (axial x lateral x elevational) speckles. The AMS filter incorporates knowledge about speckle statistics of blood and myocardium in an adaptive manner: homogeneous regions are filtered strongly, i.e. speckle noise is reduced, whereas in inhomogeneous regions the degree of filtering is low, such that transitions between blood and myocardium are preserved. The AMS filter has been proven to be effective for segmentation of echocardiographic images when using gradient based deformable models [7].

\subsection{Deformable Model}

A deformable simplex mesh model [10] was used for segmentation of the left ventricle. In this model, each vertex of the mesh $p_{i}=\left(x_{i}, y_{i}, z_{i}\right)$ is displaced in an iterative manner according to the discrete approximation of the Newtonian law of motion:

$$
p_{i+1}=p_{i}+\alpha F_{\text {int }}+F_{\text {ext }}
$$

$F_{\text {ext }}$ is an external force derived from the image data that steers the simplex mesh onto boundary structures. In this study, $F_{\text {ext }}$ consisted of an adaptive filtering based component and the newly defined maximum cross-correlation component:

$$
F_{\text {ext }}=\beta F_{\operatorname{grad}_{A M S}}+\delta F_{\text {grad }_{M C C}}+\kappa F_{\text {speed }_{A M S}}+\lambda F_{\text {speed }_{M C C}}
$$

Both AMS and MCC images were used to compute gradient and speed forces (see [11, 12 for a general description of gradient and speed forces). $F_{\text {int }}$ is a regularization force and controls the smoothness of the surface [10]. Weighting factors 

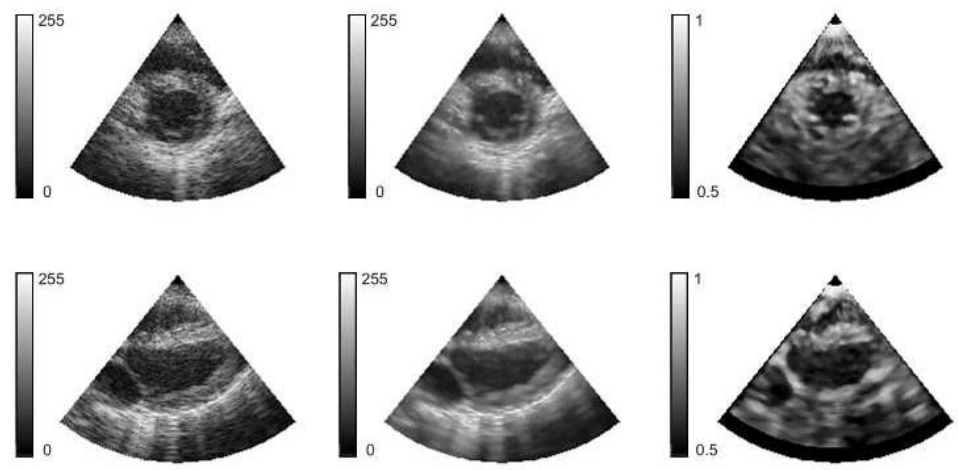

Fig. 1. Short (upper panel) and long (lower panel) axis views from a full volume dataset for the two external force types of the deformable model. Left: demodulated rf-data. Middle: data after adaptive filtering. Right: maximum cross correlation values.

$\alpha, \beta, \delta, \kappa$ and $\lambda$ were used to balance the different forces. Whereas adaptive filtering and computation of the cross-correlation was performed by processing the data along the scan-lines, computation of the external and internal forces, as well as the deformation of the simplex mesh was performed on the data in scan-converted (i.e., sector) format. Initialization of the mesh was done by interactive placement of a small spherical mesh in the center of the left ventricle.

Fig. 1 shows long and short axis views from a full volume dataset for the two external force types of the model. In this figure, the original demodulated rf-data, the data after adaptive filtering and the cross-correlation image are shown. Image data were visualized in scan-converted format, in order to obtain realistic anatomical views.

\subsection{Evaluation}

The method was evaluated by comparing left ventricular cavity contours as obtained from the segmentation method with contours obtained from manual segmentation. Papillary muscles were excluded from the left ventricular cavity. Contours were extracted from the $3 \mathrm{D}$ volume segmented by the deformable model and compared to manual segmentation for long axis (LAX) view and three short axis (SAX) cross sections (at base, mid and apical level). Three different force types were compared: using AMS force only (original model, $\beta \neq 0, \kappa \neq 0, \delta=$ $\lambda=0)$, using cross-correlation force only $(\beta=\kappa=0, \delta \neq 0, \lambda \neq 0)$, and the combined model $(\beta=\kappa=\delta=\lambda \neq 0)$. The mismatch ratio based on the Dice coefficient was computed for all three force types to the express dissimilarity between manual (Ref) and automatic segmentation $(S e g)$ :

$$
\text { Mismatch }_{\text {Dice }}=1-\frac{2(\operatorname{Re} f \cap S e g)}{(\operatorname{Ref}+S e g)}
$$




\section{Results}

3D segmentation of the left ventricle was performed in four full volume images obtained from four healthy children (6, 7, 8 and 9 years old) in the end systolic phase of the heart cycle. For each dataset, the three different force types (AMS, MCC and a combination of both AMS and MCC) were tested using the deformable model. For each dataset, the same initial position of the mesh in the center of the left ventricle was used for all three segmentation methods.

Fig. 1 illustrates that the contrast between blood and the myocardium is higher for the cross-correlation values than for the adaptive filtered data. Also endocardial regions with low echogenicity seem to have better contrast between blood and myocardium in the correlation image. Fig. 2 shows the effect of the maximum cross-correlation on the segmentation results. In this figure, segmentations of the endocardial surface in the long axis view (LAX) and three short axis views (SAX base, mid and apex) are given for an illustrative example. It can be clearly seen that for this dataset, segmentation exclusively based on the AMS data leads to overestimation of the dimension of the ventricular cavity at the apical side of the long axis view (compare mismatch ratios in Table 1, column 2, LAX). Segmentation solely based on maximum cross-correlation leads to incorrect segmentation and underestimation of the endocardial dimension, see middle panel. Combination of AMS and cross-correlation force (lower panel) improves

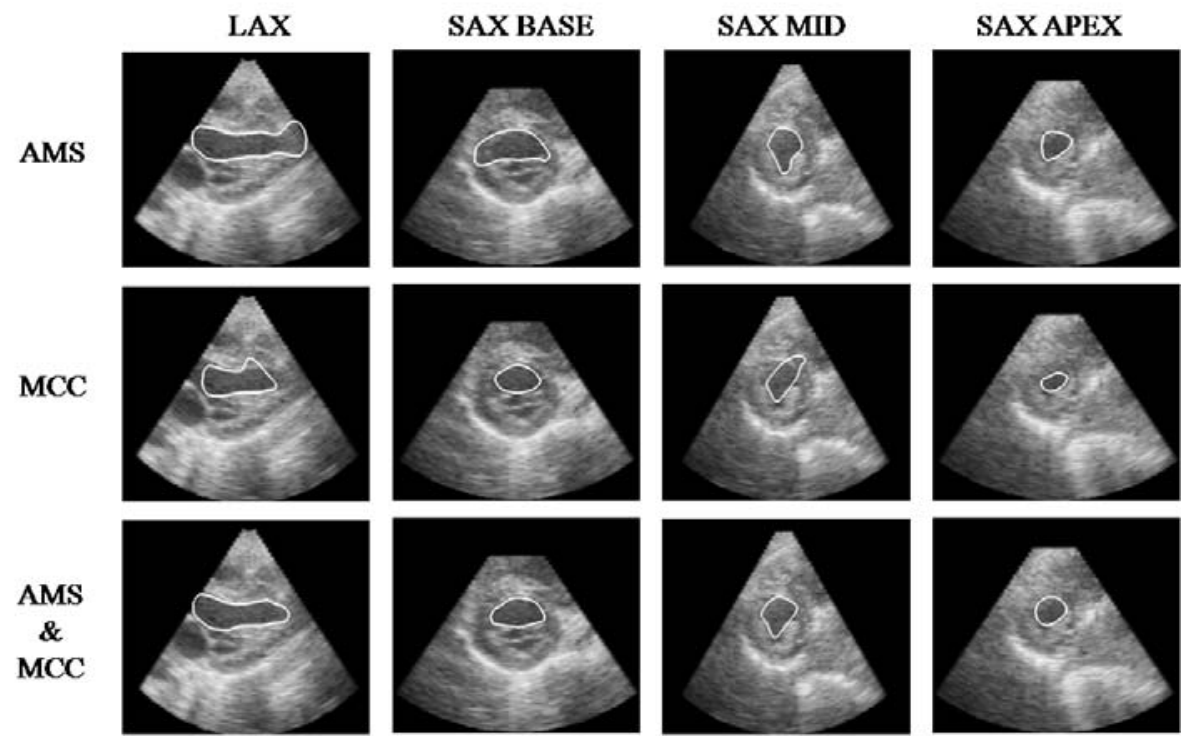

Fig. 2. Segmentation results for three settings of the deformable model for one dataset. Long and short axis views overlaid with contours of the segmented endocardial surface using AMS (upper), maximum cross correlation (MCC) (middle) and an equally weighed combination of AMS and MCC force (lower). 
Table 1. Mismatch ratios for segmentation by adaptive mean squares (AMS), maximum cross-correlation (MCC) and AMS/MCC combined methods. Long axis (LAX) view and short axis (SAX) view images for the data set of Fig. 2 .

\begin{tabular}{lcccc}
\hline $\begin{array}{l}\text { Mismatch } \\
\text { ratios }\end{array}$ & LAX & $\begin{array}{l}\text { SAX } \\
\text { Base }\end{array}$ & $\begin{array}{l}\text { SAX } \\
\text { Mid }\end{array}$ & $\begin{array}{l}\text { SAX } \\
\text { Apex }\end{array}$ \\
\hline AMS & 0.23 & 0.25 & 0.23 & 0.13 \\
MCC & 0.22 & 0.14 & 0.16 & 0.27 \\
AMS \& MCC & 0.12 & 0.14 & 0.15 & 0.12 \\
\hline
\end{tabular}

Table 2. Average mismatch ratios $(n=4)$ for segmentation by adaptive mean squares (AMS), maximum cross-correlation (MCC) and AMS/MCC combined methods for long axis (LAX) view and short axis (SAX) view images

\begin{tabular}{lcccc}
\hline $\begin{array}{l}\text { Average } \\
\text { mismatch } \\
\text { ratios }\end{array}$ & LAX & $\begin{array}{l}\text { SAX } \\
\text { Base }\end{array}$ & $\begin{array}{l}\text { SAX } \\
\text { Mid }\end{array}$ & $\begin{array}{l}\text { SAX } \\
\text { Apex }\end{array}$ \\
\hline AMS & 0.18 & 0.16 & 0.22 & 0.15 \\
MCC & 0.17 & 0.14 & 0.17 & 0.26 \\
AMS \& MCC & 0.11 & 0.12 & 0.14 & 0.13 \\
\hline
\end{tabular}

the segmentation and results in correct dimensions of the left ventricle. Also in the short axis views, combination of AMS and cross-correlation results in better segmentation of the endocardial surface. Corresponding mismatch ratios for this dataset (see Table 1) revealed that the segmentation results improved (i.e., the mismatch ratio decreased) when the cross-correlation force was added to the AMS force. Using the cross-correlation force on its own resulted in underestimation of the endocardial surface, whereas a segmentation that only used the AMS filtered data resulted in crossing boundaries in the low contrast regions. Table 2 summarizes the mismatch ratios for the four different views (LAX, SAX base, mid and apex), averaged over all four datasets. According to this table, the combination of AMS and MCC force yielded the most accurate segmentation results for all views.

To demonstrate the effect of the MCC force, the gray levels of manually drawn myocardial and blood pool regions were compared for AMS and MCC values. Figure 3 compares AMS against MCC values for myocardial regions with high and low echogenicity (apical), and a blood pool region, respectively It can be seen from this figure that the AMS force is highly distinctive in the case of blood vs. myocardial tissue with high echogenicity. However, for low contrast regions of the heart muscle, for example near the apex, blood and myocardium can no longer be classified by using the AMS values, whereas in this case, MCC still can be used to distinguish blood form myocardial tissue. 


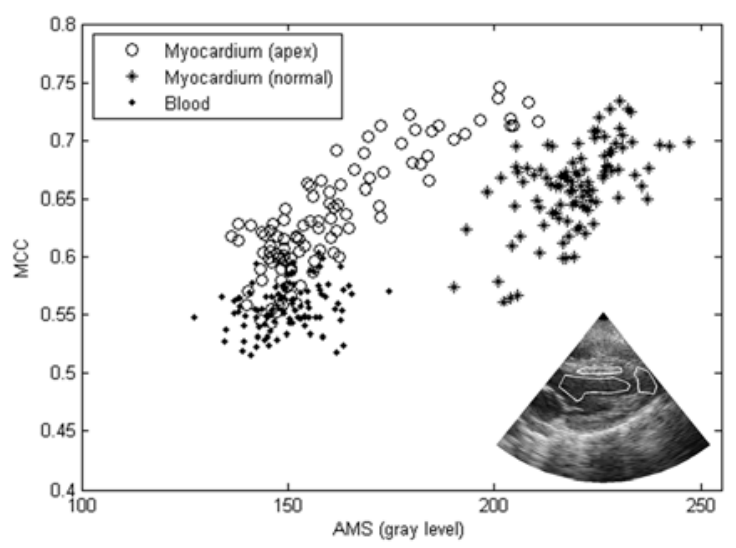

Fig. 3. Comparison between AMS (horizontal axis) and MCC (vertical axis) values for high and low contrast myocardial regions vs. blood pool region. Manually drawn regions are shown in the echogram (bottom right).

\section{Discussion and Conclusion}

In this study, we developed a method that enables segmentation of anatomical structures in the heart of children by incorporating temporal information in the model. According to these preliminary results, maximum temporal crosscorrelation values, based on the rf-signal, have additional value for the segmentation of cardiac tissue. Since correlation can still be high in areas with low echogenicity, inclusion of this parameter in the deformable simplex model as an extra feature facilitates segmentation in regions where contrast between blood pool and endocardial border is too low to perform gray-level based segmentation. This is illustrated by the proper segmentation of the apical region in Fig. 2. The complimentary character of the MCC and AMS is demonstrated in Fig. 3. In high quality images (without low contrast regions), the AMS force already yielded adequate segmentation results and the cross-correlation force did have no additional value as indicated by the mismatch ratios for AMS and the combination of MCC and AMS in the apical short axis view (Table 1).

A deformable model that only uses the cross-correlation force underestimated the blood pool region. This is most likely caused by the larger windows used in the computation of correlation values and because of the blood close to the heart wall 'adhering' to the moving heart muscle, leading to a higher correlation value, i.e., lower MCC contrast, in blood regions close to the endocardial border.

Presently, the method has been tested in the end-systolic phase where deformation of the heart is small, because with the current echo systems, the frame rate of full volume imaging is still limited in terms of the accuracy of cross-correlation values throughout the entire cardiac cycle. For example, the cross-correlation based model could be used in the end-systolic and end-diastolic phase as a more robust 
initialization for segmentation of the other frames in the cardiac cycle. In future, the method will be extended to more frames during the cardiac cycle.

Acknowledgments. This work is supported by the Dutch Technology Foundation (STW), project 06466 and Philips Medical Systems. The authors would like to thank Bob Rijk for his assistance with the echographic image acquisitions.

\section{References}

1. Noble, J.A., Boukerroui, D.: Ultrasound Image Segmentation: A Survey. IEEE Trans. Med. Imag. 25(8), 987-1010 (2006)

2. Bosch, J.G., Mitchell, S.C., Lelieveldt, B.P., Nijland, F., Kamp, O., Sonka, M., Reiber, J.H.: Automatic segmentation of echocardiographic sequences by active appearance motion models. IEEE Trans. Med. Imag. 21(11), 1374-1383 (2002)

3. Boukerroui, D., Basset, O., Baskurt, A., Giminez, G.: A multiparametric and multiresolution segmentation algorithm of 3-D ultrasonic data. IEEE Trans. Ultrason. Ferroelectr. Freq. Control 48(1), 64-77 (2001)

4. Davignon, F., Deprez, J.F., Basset, O.: A parametric imaging approach for the segmentation of ultrasound data. Ultrasonics 43(10), 789-801 (2005)

5. Dydenko, I., Friboulet, D., Gorce, J.M., D’Hooge, J., Bijnens, B., Magnin, I.E.: Towards ultrasound cardiac image segmentation based on the radiofrequency signal. Med. Image Anal. 7(3), 353-367 (2003)

6. Yan, P., Jia, C.X., Sinusas, A., Thiele, K., O'Donnell, M., Duncan, J.S.: LV segmentation through the analysis of radio frequency ultrasonic images. Inf. Process Med. Imaging 20, 233-244 (2007)

7. Nillesen, M.M., Lopata, R.G.P., Gerrits, I.H., Kapusta, L., Huisman, H.J., Thijssen, J.M., de Korte, C.L.: Segmentation of the heart muscle in 3D pediatric echocardiographic images. Ultrasound Med. Biol. 33(9), 1453-1462 (2007)

8. Chen, X., Xie, H., Erkamp, R., Kim, K., Jia, C., Rubin, J.M., O’Donnell, M.: 3-D correlation-based speckle tracking. Ultrason Imaging 27(1), 21-36 (2005)

9. Lopata, R.G.P., Nillesen, M.M., Gerrits, I.H., Thijssen, J.M., Kapusta, L., de Korte, C.L.: 4D cardiac strain imaging: methods and initial results. In: Proceedings of the IEEE International Ultrasonics Symposium, New York, U.S.A., pp. 872-875 (2007)

10. Delingette, H.: General object reconstruction based on simplex meshes. International Journal of Computer Vision 32(2), 111-146 (1999)

11. Böttger, T., Kunert, T., Meinzer, H.P., Wolf, I.: Application of a new segmentation tool based on interactive simplex meshes to cardiac images and pulmonary MRI data. Acad. Radiol. 14(3), 319-329 (2007)

12. Nillesen, M.M., Lopata, R.G.P., de Boode, W.P., Gerrits, I.H., Huisman, H.J., Thijssen, J.M., Kapusta, L., de Korte, C.L.: In vivo validation of cardiac output assessment in non-standard 3D echocardiographic images. Phys. Med. Biol. 54(7), 1951-1962 (2009) 